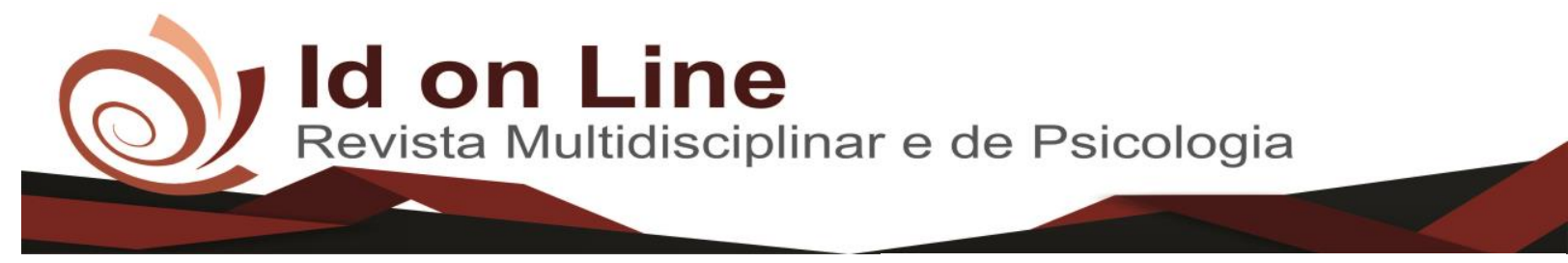

Comment

\title{
O impacto da síndrome de Moebius na saúde oral
}

\author{
Fairane Santos Soares ${ }^{1}$; Edite Novais Borges Pinchemel ${ }^{2}$
}

\begin{abstract}
Resumo: A síndrome de Moebius é uma desordem rara de caráter congênito, que se caracteriza por apresentar paralisia não progressiva dos nervos cranianos facial e abducente, em virtude da atrofia dos seus núcleos. Essa síndrome também pode afetar outros nervos cranianos com gravidade variada. Como consequência dessa paralisia, os portadores apresentam principalmente face em máscara, pouco expressiva, além de outras alterações. Ocorrem diversos comprometimentos nessa síndrome que implicam em alterações orofaciais, assim como malformações das extremidades superiores e inferiores. Sua etiologia ainda permanece obscura, mas está relacionada com a isquemia das células neurais do feto. Pela síndrome apresentar diversas implicações na saúde geral e bucal a intervenção precoce de um cirurgião-dentista é de suma importância para a qualidade de vida do paciente portador. O objetivo desse estudo é estabelecer um levantamento criterioso em bases de dados sobre a síndrome de Moebius, as complicações orais presentes e associar essas alterações a qualidade de vida de pacientes portadores da síndrome.
\end{abstract}

Palavras-chave: Síndrome de Moebius, saúde bucal, paralisia facial, qualidade de vida.

\section{The impact of Moebius syndrome on oral health}

\begin{abstract}
Moebius syndrome is a rare congenital disorder characterized by non-progressive facial and abducent cranial nerve palsy due to the atrophy of its nuclei. This syndrome can also affect other cranial nerves with varying severity. As a consequence of this paralysis, the patients present mainly mask face, not expressive, besides other alterations. There are several compromises in this syndrome that imply orofacial alterations, as well as malformations of the upper and lower extremities. Its etiology remains unclear, but is related to the ischemia of the neural cells of the fetus. Because the syndrome presents several implications in general and oral health, the early intervention of a dental surgeon is of paramount importance for the quality of life of the patient. The objective of this study is to establish a careful survey of Moebius syndrome databases, the oral complications present and to associate these changes with the quality of life of patients with the syndrome.
\end{abstract}

Key words: Moebius syndrome, oral health, facial paralysis, quality of life.

\section{Introdução}

A síndrome de Moebius foi descrita pela primeira vez por Von Graefe em 1880, mais tarde, em 1992, Paul Julius Möbius estudou 43 casos de paralisia dos nervos do crânio, observando a

\footnotetext{
${ }^{1}$ Graduanda em Odontologia pela FAINOR. E-mail: fairane.soares@ gmail.com

${ }^{2}$ Mestre e doutoranda em Odontopediatria pelo Centro de Pesquisa São Leopoldo Mandic

E-mail: editenbpinchemel@gmail.com
} 
presença de paralisia total ou parcial dos nervos abducente e facial e, além disso, associou essa alteração com outras malformações, sendo assim, a síndrome passou a receber o seu nome, Moebius $^{1-3}$.

A síndrome é uma desordem neurológica rara, congênita e não progressiva, de severidade variada que se caracteriza por apresentar principalmente paralisia, geralmente bilateral, do nervo facial (sétimo par de nervos cranianos), podendo também afetar outros nervos cranianos como o abducente, estruturas da face e orais, além de apresentar, possivelmente, malformação no sistema responsável pelas emoções e comportamentos sociais (límbico). Essa alteração resultará num comprometimento da expressão facial, apresentando a face em máscara ${ }^{4-6}$.

Diversas são as manifestações orofaciais decorrentes da síndrome de Moebius, como hipoplasia dentária, cárie, doença periodontal, úvula bífida, dificuldade no momento de execução de movimentos excursivos das maxilas, ausência de tônus dos músculos da face e também da língua, dentre outros ${ }^{5}$.Existem também alterações resultantes da síndrome como a malformação das extremidades superiores e inferiores, como a união de dois ou mais dedos dos pés ou das mãos, além do pé torto congênito ${ }^{7}$.

Com uma grande dificuldade de se determinar o diagnóstico da síndrome de Moebius, em 2007 um grupo de médicos e pesquisadores da fundação "Síndrome de Moebius" se reuniram para criar critérios mínimos para ajudar no diagnóstico da síndrome clássica. Para isso o paciente deve apresentar paralisia facial congênita uni ou bilateral, que não seja progressiva, além de possuir uma abdução dos olhos dificultada ${ }^{8,9}$. Já para Kumar, o indivíduo deve apresentar dificuldade fonética e na deglutição; hipoplasia de língua; alterações orofaciais e musculoesqueléticas; paralisia total ou parcial do nervo facial associada ou não com o nervo abducente, oculomotor e troclear; malformação dos membros ${ }^{10}$. Portanto, o diagnóstico é determinado ainda quando criança, observando o fechamento incompleto da pálpebra quando o indivíduo está dormindo, falta de sorriso e mímica facial, e dificuldade de sucção ${ }^{10,11,3}$. Em virtude da dificuldade de fechar o diagnóstico da síndrome, e da ampla ocorrência de alterações no indivíduo, existem diagnósticos diferenciais, influenciando na determinação do correto diagnóstico 9 .

Estima-se a prevalência em 1/250000 nascidos vivos, igual para ambos os sexos e com $2 \%$ para casos com antecedentes familiares do total documentados ${ }^{3}$. 
Sua etiologia ainda permanece obscura. A hipótese mais disseminada e aceita é a causa gerada pela isquemia/hipóxia das células nervosas presentes no feto, consequente da parada do fluxo sanguíneo durante os três primeiros meses de gestação. Isso pode ser causado por uma contração uterina em decorrência do uso de fármacos ou substâncias tóxicas. Estudos apontam a suposta associação entre o uso do misoprostol e a síndrome de Moebius, uma alteração rara. O misoprostol é uma substância desenvolvida para tratamento e prevenção de úlcera gastroduodenal, que começou a ser usada ilegalmente para provocar o aborto, pois estimula contrações e o alargamento do colo do útero. Outras possíveis causas para a síndrome são: rubéola durante gravidez, diabetes gestacional, aumento da temperatura corporal, distúrbio no desenvolvimento vascular romboencefálico, causado por alteração genética e relações consanguíneas ${ }^{3,10-16}$.

Em virtude da presença da síndrome esses pacientes apresentam sérios problemas no quesito emocional ${ }^{17}$. Ressalta-se a importância do cirurgião-dentista no atendimento precoce desses pacientes, para que as alterações orais presentes no indivíduo sejam tratadas a partir de um planejamento integral ${ }^{4}$, estabelecendo um protocolo preventivo para patologias orais como a cárie e a doença periodontal.

O objetivo desse estudo é comentar os possíveis impactos na saúde oral de indivíduos portadores da síndrome de Moebius a partir de uma revisão de uma série de artigos das principais bases de dados.

\section{Revisão de Literatura}

A síndrome de Moebius, de acordo com Escoda-Francolí, Sánchez-Garcés e GayEscoda (2009) é uma alteração neurológica não progressiva que envolve principalmente os nervos cranianos VI e VII, podendo ser uni ou bilateral, resultando em uma paralisia facial que alterará a mímica facial do indivíduo ${ }^{14}$. As principais alterações geradas por essa paralisia é a face em máscara, apresentando pouca expressão facial, além de outras alterações no sistema musculoesquelético ${ }^{18-23}$.

A etiologia da síndrome de Moebius ainda permanece indefinida, com diversas teorias de acordo com os artigos consultados. Para Corrêa; Mastrella (2012) ${ }^{12}$ e Matanna e 
colaboradores $(2010)^{24}$, existem duas vertentes que hipotetizam a causa da síndrome, a ambiental e a genética. Na questão ambiental está o uso de álcool, drogas como a cocaína, psicofármacos, exposições a infecções, diabetes gestacional, hipertermia, uso de talidomida e o misoprostol no primeiro trimestre da gravidez $z^{4,12,16,24-26}$. Já na questão genética é mencionado o cromossomo x ou também chamado de mutação da banda q12.2 do cromossomo 13 do cariótipo humano $^{12,24}$. A teoria mais aceita é a isquemia transitória fetal ${ }^{15,27-30}$.

O misoprostol é um análogo da prostaglandina E, que está sendo utilizado como abortivo ilegalmente, sendo um dos fatores associados à etiologia da síndrome de Moebius por teoricamente afetar a contratilidade uterina e a irrigação de sangue no feto, gerando uma hipóxia e isquemia do nervo facial ${ }^{24,26,30,31}$.

É possível observar a presença da síndrome logo após o nascimento da criança, e pode ser diagnosticada a partir de características como a inabilidade de sucção, o não fechamento completo das pálpebras durante o sono, acúmulo de saliva na comissura labial, inexpressão facial e ausência de sorriso mesmo no instante em que a criança chora ${ }^{3-5,10,11,25,32}$.

Em se tratando das manifestações orofaciais dos pacientes portadores da síndrome de Moebius pode-se afirmar que são comuns e diversas, além de afetarem diretamente a qualidade de vida desses indivíduos ${ }^{33}$. Essas alterações se apresentam como lesões cariosas, doenças no periodonto, hipoplasia dentária, sialorreia, falta de selamento dos lábios, fissuras na língua, tônus muscular facial e lingual diminuídos, micrognatia, microstomia, úvula bífida, fissura palatina, mordida aberta, dificuldade em fazer movimentos da mandíbula, fissuras nas pálpebras, ptose palpebral, deformidade da orelha com ou sem perda da audição ${ }^{5,13-16}$.

A implicação da síndrome de Moebius na saúde oral da criança é ampla e bastante complexa. De acordo com Scarpelli e colaboradores (2008) ${ }^{11}$, existe uma dificuldade eminente no tratamento desses pacientes em virtude da limitação da abertura de boca e da falta de cooperação da maioria dos indivíduos ${ }^{5,11}$. Além disso, existe a possibilidade dos músculos periorais e a língua estarem pouco desenvolvidos levando também a uma higiene precária $^{4,16,28,34}$. Com isso a cavidade oral fica predisposta a apresentar o desenvolvimento de patologias causadas pela falta ou má realização da higiene oral como a cárie e a doença periodontal.

Uma explicação para a grande quantidade de lesões cariosas em crianças com a síndrome de Moebius é o uso da mamadeira noturna segundo ${ }^{4,35}$. Geralmente esses pacientes 
apresentam retardo cognitivo ou falta de coordenação motora e isso facilita a higienização precária da cavidade oral ${ }^{5,37}$. Em virtude da presença de características como face em máscara, acúmulo de saliva na comissura labial, dificuldade ao falar e estrabismo, podem levar a um diagnóstico diferencial de retardo mental ${ }^{4,10}$. Esses pacientes podem apresentar uma malformação do palato, além de outros fatores como pouca mobilidade da língua e da mandíbula causando um problema na ingesta e deglutição de alimentos e líquidos ${ }^{6,25,38,39}$.

Em relação a hipoplasia do esmalte, alteração que pode ser encontrada em pacientes com síndrome de Moebius, podem ser áreas facilitadoras para instalação do biofilme dental, tornando os elementos dentários suscetíveis a lesões cariosas. Por isso um manejo preventivo primeiramente e possivelmente procedimentos restauradores se fazem necessários ${ }^{7,23,34,37}$.

Para esses pacientes, enfatiza-se a necessidade de controles periódicos frequentes, geralmente de 3 em 3 meses, além do uso de produtos como clorexidina e flúor tópico. É de suma importância também a orientação e motivação quanto a higiene oral correta e bem-feita, de preferência realizada por um adulto responsável ${ }^{4}$. Durante os procedimentos também é aconselhado usar vaselina sólida nas mucosas labiais, em virtude do ressecamento labial nesses pacientes, para não causar desconforto e lesões nos tecidos ${ }^{4,34}$.

Crianças com a síndrome em questão, podem manifestar problemas com a fala, em decorrência de alterações como a dificuldade de fechar a boca, ausência de movimentos de alguns músculos da face, hipersalivação, perda de elementos dentários, malformação da língua e palato, micrognatia e dentre outros ${ }^{32}$. De acordo com Freitas e colaboradores $(2006)^{4}$, os pacientes que apresentam a síndrome mostram dificuldade nos sons das letras $\mathrm{m}, \mathrm{b}, \mathrm{f}$ e $\mathrm{p}$, além da dificuldade na comunicação entre pessoas, levando o indivíduo a ler lábios para compensar o problema com a fala 4 .

Em virtude das malformações geradas pela síndrome, estas vão acometer diretamente na qualidade de vida desses indivíduos. Geralmente em decorrência da face em máscara e a falta de expressões faciais, esses pacientes tem baixa autoestima e afeta a imagem do indivíduo. Assim sendo, a presença da síndrome e suas alterações podem levar a um desajustamento social e emocional do indivíduo. Prova-se a partir de comportamentos como inibição, introversão, sentimento de ser inferior a outras pessoas, além de desagrado com a vida e pouca expectativa do futuro ${ }^{40-42}$. 
É fundamental apresentar ao portador da síndrome e ao responsável a importância da higiene oral e os problemas causados pela falta da mesma ${ }^{5,43}$. Além disso, é interessante que o profissional que se dispuser atender pacientes com síndrome de Moebius, esteja ciente de todas as características que a síndrome pode apresentar e conhecer os limites e as necessidades do paciente ${ }^{4}$.

\section{Conclusões}

Mediante um estudo a respeito da síndrome de Moebius e a sua implicação na saúde oral dos pacientes portadores, chegou-se à conclusão que muito pouco foi publicado sobre o assunto. Portanto, há uma necessidade de mais estudos a respeito da síndrome de Moebius, da sua relação com a conduta e os cuidados profissionais para com esses pacientes visando uma melhora na saúde oral deles. Tendo em vista que quanto mais precoce for o diagnóstico da síndrome e também com o tratamento das alterações orais presentes na criança serão melhores as chances de uma boa qualidade de vida.

\section{Referências}

1. Vasconcelos GC, da Silva FBD, de Almeida HC, Boas MLMV, Álvares MG. Síndrome de Möbius: achados clínicos e cirúrgicos em 7 pacientes. Arq Bras Oftalmol. 2001;64(3):2115.

2. Palmer-Morales Y, Zárate-Márquez RE, Prince-Vélez R, González-Méndez R, ZamarripaSandoval TA, Verdugo-Salazar N, et al. Síndrome de Moebius: informe de un caso clínico. Rev Med Inst Mex Seguro Soc. 2013;51(5):584-6.

3. Picciolini O, Porro M, Cattaneo E, Castelletti S, Masera G, Mosca F, et al. Moebius syndrome: clinical features, diagnosis, management and early intervention. Ital J Pediatr. 2016;42(1):56.

4. Freitas AC, Nelson-Filho P, de Queiroz AM, Assed S, Silva FWGP. Síndrome de Moebius: relato de caso clínico. Rev Odontol UNICID. 2006;18(3):297-302. 
5. Serra AVP, Moreira CVA, de Azevedo RA, Santos ND, Silva LOR. Síndrome de Moebius em paciente com fissura labiopalatina: relato de caso. RBO. 2017;8(4):125-31.

6. Saconato M, Guedes ZCF. Estudo da mastigação e da deglutição em crianças e adolescentes com sequência de Möbius. Rev Soc Bras Fonoaudiol. 2009;14(2):165-71.

7. Vecchio RMSD, Morales-Chávez MC. Manejo odontológico del síndrome de Moebius. Rev odontopediatr latinoam. 2016;6:118-25.

8. Carta A, Mora P, Neri A, Favilla S, Sadun AA. Ophthalmologic and systemic features in möbius syndrome an italian case series. Ophthalmology. 2011; 118(8):1518-23.

9. MacKinnon S, Oystreck DT, Andrews C, Chan WM, Hunter DG, Engle EC. Diagnostic distinctions and genetic analysis of patients diagnosed with Moebius syndrome. Ophthalmology. 2014; 121(7):1461-8.

10. Kumar D. Moebius syndrome. J Med Genet. 1990; 27(2):122-6.

11. Scarpelli AC, Vertchenko TB, Resende VLS, Castilho LS, Paiva SM, Pordeus IA. Möbius syndrome: a case with oral involvement. Cleft Palate Craniofac J. 2008; 45(3):319-24.

12. Corrêa MCDV, Mastrella M. Aborto e misoprostol: usos médicos, práticas de saúde e controvérsia científica. Ciên Saúde Colet. 2012; 17(7):1777-84.

13. Bianchi B, Copelli C, Ferrari S, Ferri A, Sesenna E. Facial animation in children with Moebius and Moebius-like syndromes. Int J Oral Maxillofac Surg. 2010; 39(11):1066-73.

14. Escoda-Francolí J, Sánchez-Garcés MA, Gay-Escoda C. Oral implant rehabilitation in a patient with Moebius syndrome. Med Oral Patol Oral Cir Bucal. 2009; 14(6):E295-8.

15. Fontenelle L, Araújo APQC, Fontana RS. Síndrome de Moebius: relato de caso. Arq Neuro Psiquiatr. 2001; 59(3-B):812-4.

16. Rizos M, Negrón RJ, Serman N. Möbius syndrome with dental involvement: a case report and literature review. Cleft Palate Craniofac J. 1998; 35(3):262-8.

17. Briegel W. Psychopathology and personality aspects of adults with Möbius sequence. Clin Genet. 2007; 71(4):376-7.

18. Gonzalez CH, Marques-Dias MJ, Kim CA, Sugayama SMM, Da Paz JA, Huson SM, et al. Congenital abnormalities in Brazilian children associated with misoprostol misuse in first trimester of pregnancy. Lancet. 1998; 351(9116):1624-6.

19. Nunes ML, Friedrich MAG, Loch LF. Association of Misoprostol, Moebius syndrome and congenital central alveolar hypoventilation: case report. Arch Neuropsychiatry. 1999; 57(1):89-91. 
20. Boudoux DD, Matos MAG, Gonçalves ED, Rocha M, Ventura LO, Hinrischen SL, et al. Síndrome de Moebius relacionada à ameaça de abortamento. Rev Bras Oftalmol. 2000; 59(3):173-7.

21. Cronemberger MF, Moreira JBC, Brunoni D, Mendonça TS, Alvarenga EHL, Rizzo AMPP, et al. Ocular and clinical manifestations of Moebius syndrome. J Pediatric Ophthalmol Strabismus. 2001; 38(3):156-62.

22. Sensat ML. Mobius syndrome: a dental hygiene case study and review of literature. Int J Dent Hyg. 2003; 1(1):62-7.

23. Brandão CB, de Queiroz AM, Nelson-Filho P, Brandão RB, Silva FWGP, Díaz-Serrano $\mathrm{KV}$. Aspectos odontológicos en paciente portador del síndrome de Moebius: relato de caso. Acta odontol venez. 2007; 46(3).

24. Matanna MC, Lucena LF, Ribeiro FE, Bortagaray S, Teles AR. Síndrome de MoebiusPoland: relato de caso e revisão bibliográfica. Rev AMRIGS. 2010; 54(2):197-201.

25. Franco RM, Motta CM, Pereira GAF, Santalla TP, Franco NM, Vieira MW, et al. Sequência de Moebius: relato de caso e revisão de literatura. Rev Fac Ciênc Méd Sorocaba. 2011; 13(1):26-8.

26. Almeida MBV, Brandt CT, Lima JA, Rolim Filho EL, Malheiros Jr HJA, Van der Linden $\mathrm{V}$, et al. A incidência de anomalias ortopédicas em portadores de Sequência de Möbius e sua associação com o uso do misoprostol. Rev Bras Ortop. 2007; 42(1/2):10-6.

27. Volpe J. Neurology of the neyborn. $3^{\text {a }}$ ed. Philadelphia: Saunders, 1995.

28. Dotti MT, Federico A, Palmeri S, Guazzi GC. Congenital oculo-facial paralysis (Moebius syndrome): evidence of dominant inheritance in two families. Acta Neurol (Napoli). 1998; 11(6):434-8.

29. Silva MBS, Ferreira AS, Habr SF, Bernardi MM. Misoprostol and teratogenesis in neonates. Braz J Pharm Sci. 2009; 45(3):417-22.

30. Diogo FSF, Aguiar SMHCA, Faria CCM, Rocha GST. Utilização do misoprostol (Cytotec (B) como droga abortiva e sua relação com a síndrome de Moebius: relato de caso. Rev odontol Araçatuba. 2009; 30(1):31-5.

31. Bandim JM, Ventura LO, Miller MT, Almeida HC, Costa AES. Autism and Möbius sequence: an exploratory study of children in northeastern Brazil. Arq neuro-psiquiatr. 2003; 61(2A):181-5.

32. Alves GAS, Lima ILB, Lima JAS, Lucena BTL, Delgado IC. Alterações de fala em crianças com síndrome de Möbius: análise da literatura. Revista do GEL. 2015; 12(1):33-45. 
33. Shashikiran ND, Subba Reddy VV, Patil R. "Moebius syndrome": a case report. J Indian Soc Ped Prev Dent. 2004; 22(30):96-9.

34. Serpa-Pinto MV, de Magalhães MH, Nunes FD. Moebius syndrome with oral involvement. Int J Pediatr Dent. 2002; 12(6):446-9.

35. Lin KJ, Wang WN. Moebius syndrome: reporte of case. ASDC J Dent Child. 1997; 64(1):64-7

36. Mendoza-Urbano DM, Ramírez-Cheyne J, Saldarriaga-Gil W. Piebaldismo-Moebius y exposición prenatal a misoprostol: reporte de un caso. Iatreia. 2016; 29(1):81-7.

37. Ha CY, Messieha ZS. Management of a patient with Mobius syndrome: a case report. Space Care Dentist. 2003; 23(3):111-6.

38. Jones KL. Smith padrões reconhecíveis de malformações congênitas. $5^{\text {a }}$ ed. São Paulo: Manole; 1999.

39. Marambaia O, Gomes A, Marambaia P, Araújo AHB, Pimentel K, Lima MP, Almeida F. Síndrome de Moebius associada a Misoprostol. Acta ORL. 2008; 26(3):169-72.

40. Bogart KR, Matsumoto D. Living with Moebius syndrome: adjustment, social competence, and satisfaction with life. Cleft Palate Craniofac J. 2010; 47(2):134-42.

41. Broussard AB, Borazjani JG. The faces of Moebius syndrome: recognition and anticipatory guidance. MCN Am J Matern Child Nurs. 2008; 33(5):272-8.

42. Fernandes SD, Ferreira ITG, Moreira JC, Mendonça ARA. Síndrome de Möbius: significado na vida dos portadores. Rev Soc Bras Clin Med. 2015; 13(1):2-9.

43. Nassbaum BL. Dental care for patients who are unable to open their mouths. Dent Clin C Am. 2009; 53(2):323-8.

\section{Como citar este artigo (Formato ABNT):}

SOARES, Fairane Santos; PINCHEMEL, Edite Novais Borges. O impacto da síndrome de Moebius na saúde oral. Id on Line Rev.Mult. Psic., 2018, vol.12, n.42, Supl. 1, p.66-74. ISSN: 1981-1179.

Recebido: 29/10/2018.

Aceito: 01/11/2018 